\title{
A NOTE ON CZECH PRONUNCIATION
}

Since there are many Czech names in this book, some indication as to how to pronounce them may be helpful. Written Czech is mostly phonetic, and (unlike in English) letters are pronounced consistently in the same way wherever they occur. Diacritical marks either lengthen the vowel (as in a, á) or change the sound altogether (as in $c$, č). Stress is usually on the first syllable of a word.

The following is a rough guide: other letters are sounded more or less as they are in English.

$\mathrm{a}$ is between the a in bat and the $u$ in but

á is like the a in car

$\mathrm{c}$ is like the ts in bats

$\check{c}$ is like the ch in church

$d$ is like the $d$ in dune

$\mathrm{e}$ is like the $\mathrm{e}$ in end

é is like the ea in pear

è is like the ye in yet

ch (treated as a single letter) is like the ch in the Scottish loch

$i$ is like the $i$ in bit

$i$ is like the ee in beet

$\mathrm{j}$ is like the $\mathrm{y}$ in yet, never like the English $\mathrm{j}$ as in jet

n is like the ni in onion

$\mathrm{q}$ is pronounced $\mathrm{kv}$

$r$ is rolled, as in Scottish English

r has no English equivalent; it sounds like a combination of a rolled $r$ and the sound $\check{z}$, as in the name of the composer Antonín Dvořak

$\check{s}$ is like the sh in ship

$\mathrm{t}$ is like the $\mathrm{t}$ in tune

$\mathrm{u}$ is like the oo in foot

ú and $\mathrm{u}$ are like the oo in moon

$\mathrm{w}$ is pronounced like an English $\mathrm{v}$

$y$ is identical in sound to $i$

$y$ is identical in sound to 1

$\tilde{z}$ is like the $s$ in leisure

When followed by $i$ or $\hat{1}$, the letters $d, n$, and $t$ are pronounced like $\AA$, $\check{n}$, and $\mathfrak{t}$.

The dipthong ou combines the Czech $o$ and $u$ in sequence, sounding something like the oa combination in boat, not like the ou in round or ounce.

As a rule, all letters in Czech are sounded separately (an exception is the $j$ in jsem, meaning "I am," which is seldom vocalized). The name Palacký is thus pronounced Pal-ats-kee, not Pal-a-kee. 
